\title{
PREMIÈRES OBSERVATIONS SUR LE COMPORTEMENT DES INSECTES POLLINISATEURS DE LA LUZERNE
}

\author{
IMAR
}

\author{
J. LECOMTE \\ Station de Recherches Apicoles \\ Bures-sur-Yvette.
}

Le problème de la pollinisation de la Luzerne par les insectes ne s'était pas posé avec acuité, en France, jusqu'à ces dernières années.

Il semble que la situation ait rapidement évolué, au moins dans la moitié Nord du pays, et que les baisses importantes de rendement récemment enregistrées puissent, du moins partiellement, dépendre d'une carence en insectes pollinisateurs.

Dans cette perspective, l'Institut National de la Recherche Agronomique a envisagé d'étudier les possibilités d'utiliser l'Abeille domestique conme agent pollinisateur.

S'il est trop tôt pour parler des essais déjà effectués et s'il est sûrement nécessaire d'attendre plusieurs années avant de porter un jugement définitif sur l'intérêt de l'Abeille en Irance, du moins est-il possible de présenter quelques résultats relatifs au comportement des insectes pollinisateurs.

\section{Néthodes de trarail.}

Il sera bon pour commencer de rappeler à quel point l'année I959 et surtout la période de floraison de la I tuzerne à graine a été exceptionnelle. J'ai étudié la faune pollinisatrice de 52 champs situés dans les départements de la Seine-et-Oise, Fure-et-I,oir, I oiret, Loir-et-Cher, Indre-et Loire, Maine-et-I, oire, Vendée et Manche. Toute cette région a été caractérisée par un climat " exceptionnellement sec, chaud et ensoleillé " pour reprendre les termes du résumé mensuel du temps publié par la Direction de la Météorologie Nationale.

Ces conditions ne permettent évidenment pas de généraliser les observations effectuées cette année et tout porte à croire qu'en année humide les résultats des observations auraient été tout autres. . 
Le premier point étudié est la présence et la densité des Abeilles butineuses sur le champ de Luzerne.

$\mathrm{L}_{\mathrm{L}}$ méthode utilisée est très simple: une bande de longueur variable et d'une largeur de un mètre cinquante est minutieusement prospectée à travers le champ. Le nombre d'Abeilles au travail est noté et par la suite ramené au mètre carré.

La longueur de la bande est, comme je l'ai dit, assez variable. Dans la pratique avec un assez grand nombre de butineuses, je me suis en général contenté d'un comptage effectué sur une bande de 20 à 30 mètres. Si le nombre des butineuses était réduit, j'effectuai le comptage sur des bandes de roo mètres et plus.

Sur les 52 champs étudiés, 9 seulement n'étaient pas visités. La densité maximum observée dans des conditions normales, c'est-à-dire sans présence d'un rucher à proximité immédiate fut de 5 butineuses au mètre carré.

La densité moyenne pour l'ensemble des 4I champs visités par des abeilles est de 0,50 butineuse au mètre carré. Les champs étıdiés présentaient naturellement un assez grand nombre de différences, par exemple : densité de pieds au mètre carré, nombre de fleurs, etc. Dans tous les cas cependant j'ai choisi pour ces observations prélininaires des champs présentant un certain nombre de caractères communs. A savoir : état de pleine floraison, superficie au moins égale à r 500 mètres carrés, absence de rucher à proximité inmédiate, c'est-à-dire environ 500 mètres, absence d'autres plantes mellifères fleuries à proxinité.

D'autre part les observations ont toutes été effectuées entre 8 heures et I 8 heures, et diverses variables météorologiques ont été notées : vitesse du vent, température de l'air, nébulosité etc. Sans d'ailleurs qu'un de ces facteurs, dans les limites des variations observées, puisse étre nis en relation avec les variations de densité en butineuses.

Ces caractères généraux pouvant être exclus, à quoi peuvent se rapporter les différences constatées entre les densités en butineuses. Il est bien entendu que mes méthodes étant très grossières je ne m'attarderai qu'aux différences très importantes.

\section{Premiers résultats.}

Un premier point paraît établi, celui d'une corrélation positive entre la taille movenne des pieds de luzerne d'un champ et le nombre observé d'Abeilles butineuses.

I e tableau ci-joint illustre clairement cette tendance d'après des résultats numériques établis sur 3,1 champs.

Il est bien évident que le facteur " taille "n'est pas à incriminer en lui-mênne, mais qu'il est en corrélation arec un facteur attractif qui reste 


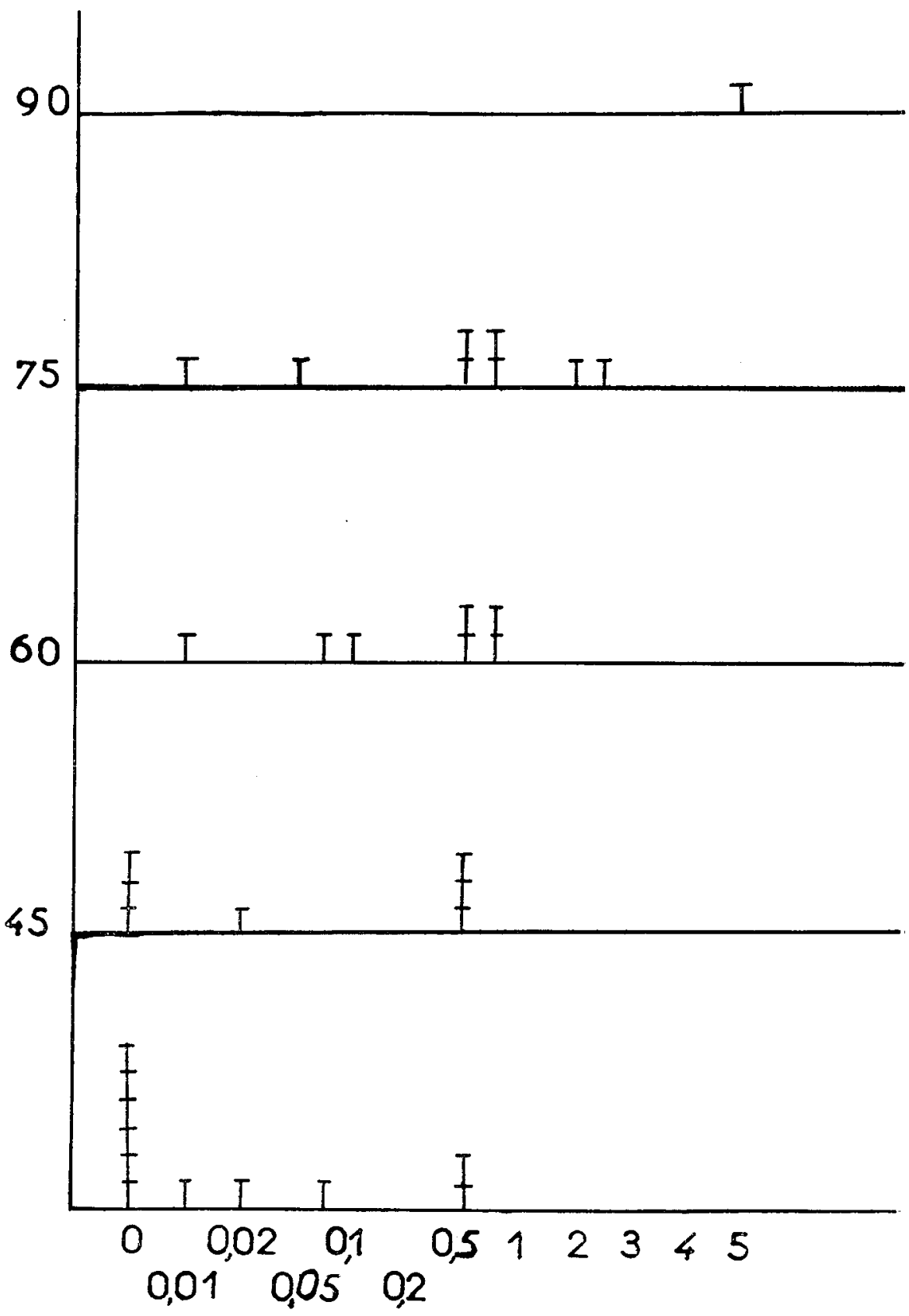

FIG. I. - En ordonnée: Hauteur moyenne des pieds de Luzerne en centimitres. - En abscisse: Nombre moyen de butineuses au mètre carré. Chaque I représerıte un chanp. I.es moyennes respectives sont de $0,09-0.2 x-0.36-0.87-5$. 
à déterminer. Il est très significatif que des champs " versés " que je n'ai pas inclus dans le tableau aient eu un taux de visite très élevé.

Un deuxième point paraît également établi, il s'agit du rapport entre l'humidite du sol et le nombre de butineuses présentes sur les fleurs.

La simple observation permet d'affirmer que ce facteur rentre certainement en ligne de compte.

Une première comparaison a été effectuée dans le marais vendéen. Dans la région comprise entre St-Jean-de-Monts et Bouin, les champs sont très généralement entourés de fossés.

Deux catégories ont été établies, celle des champs entourés de fossés très secs et celle des champs entourés de fossés pleins d'eau.

Les champs secs, au nombre de 6 , ont une densité moyenne en butineuses de 0,02 au $\mathrm{m}^{2}$, tandis que les champs humides au nombre de 4 ont une densité moyenne de 0,7 au $\mathrm{m}^{2}$.

Ces champs sont parfois distants d'une centaine de mètres à peine, ils ont été étudiés aux mêmes heures et dans des conditions strictement comparables.

Un exemple plus spectaculaite est celui d'une observation faite en Indre-et-Loire. J'ai effectué des comptages dans trois champs situés sur le sommet d'un coteau calcaire aux environs immédiats de Chinon et j'ai constaté que la densité moyenne en Abeilles butineuses était en moyenne de o,or au $\mathrm{m}^{2}$.

Dans le même temps, dans la plaine alluviale située entre Vienne et Loire et seulement à trois kilomètres de distance, la densité atteignait 3,I au mètre carré pour les trois champs étudiés. Enfin dans le département de la Manche, trois champs situés sur les falaises dominant la baie du Mont Saint-Michel se sont révélés absolument dépourvus de butineuses, bien qu'il soit possible de trouver des abeilles dans les environs. Par contre le seul champ que j'ai rencontré en état de floraison suffisamment avancé, dans les Polders, à quelques kilomètres de distance, présentait une densité en butineuses de 0,43 an $\mathrm{m}^{2}$.

Le nombre de butineuses au mètre carré n'est pas le seul facteur qui rentre en ligne de compte pour évaluer l'activité pollinisatrice de l'Abeille.

I1 faut également connaitre la vitesse de trazail, c'est-à-dire le nombre de fleurs visitées à la minute et le pourcentage de déclenchements effectués. Je rappelle que l'insecte n'opère la pollinisation que s'il déclenche le relèvement de la colonne sexuelle de la fleur de Luzerne.

En ce qui concerne la vitesse de travail, l'observation de I 139 visites domne une durée moyenne de 43 secondes pour Io visites, la butineuse la plus rapide ayant effectué ces dix visites en 22 secondes et la plus lente en go secondes.

Le taux de déclenchement n'a pu être calculé que sur 773 visites. En effet, il est impossible d'effectuer des observations correctes si les fleurs 
ne sont pas parfaitement immobiles et le plus léger vent interdit de suivre avec suffisamment de précision le comportement de l'abeille butineuse.

Par ailleurs, le taux de déclenchement est calculé par rapport au nombre total des visites, sans que je me sois préoccupé de distinguer les visites très courtes de celles comportant un butinage effectif ou les visites de fleurs déjà déclenchées. Dans ces conditions, j'ai pu observer I3 déclenchements sur 773 visites ce qui donne un tatı de déclenchement d'environ I, 6 p. roo pour l'ensemble des butineuses.

Ce taux est d'ailleurs relativement élevé comme il est possible de le voir en consultant le tableau que j'ai donné dans une revue publiée antérieurement (LECOMTE, I959).

Certaines butineuses semblent plus particulièrement disposées à effectuer le déclenchement. Sur les 8 butineuses aỹant effectué le déclenchement, une l'a effectué 3 fois pour rot visites et une autre 3 fois pour 76 visites. Entin une des très rares butineuses de pollen que j'ai pu apercevoir a opéré 2 déclenchements en 3 visites arant de s'envoler hors de ma vue.

\section{Eirionaté du hutinage ; principes de mesures.}

'Trois données : nombre de visites à la minute, taux de déclenchement et nombre de fleurs au mètre carré peurent permettre de calculer approximativement l'efficacité d'une population de butineuses. De 25 à 50 heures/abeille semblent ainsi nécessaires pour polliniser un mètre carré si l'on admet un chiffre moyen de foo fleurs au mètre carré, un taux de déclenchement compris entre I et 2 p. Ioo et tune vitesse de travail de 43 secondes pour ro fleurs.

Dans ce calcul, nous négligeons le fait que les fleurs sont butinées au hasard et non pas systématiquement l'une après l'autre. Ceci n'intervient pas dans le calcul du taux de déclenchement, puisqu'il s'agit de cliffres observés, mais on ne pent évidemment déduire du nombre de visites à la minute le laps de temps nécessaire pour visiter les foo fleurs d'un mètre carré.

'Tout ce que l'on peut faire, c'est d'évaluer grossièrement que clans les meilleures conditions, avec nne densité en butineuses de 0,5 an mètre carré, de 4 à 8 jours de travail seront nécessaires pour obtenir une pollinisation satisfaisante. La durée de vie moyenne d'une fleur de luzerne étant nettement inférieure l'on se rend compte qu'il ne peut I aroir de pollinisation complète avec une aussi faible densité en butineuses.

\section{Pollinisateurs auters que l'Abrille.}

Mais les pollinisateurs de la luzerne ne peuvent se laisser réduire à la seule Abeille domestique, de nombreux autres Hýménoptères doivent être pris en considération. 
Remarquons tout de suite que cette faune est très pauvre par rapport aux populations d'Abeilles signalées.

29 champs sur les 52 étudiés ne comportaient aucun hyménoptère sauvage visible et la densité moyenne des autres champs atteint seulement 0,02 individus au mètre carré. I, champ le plus riche, à cet égard, situé aux environs de Montfaucon, Maine-et-I,oire, avait une densité de $0, I_{5}$ individus au mètre carré.

La vitesse de travail des différentes espèces rencontrées est assez voisine de celle de l'Abeille.

J'ai ainsi trouvé des valeurs moyennes de :

42 secondes pour Io visites pour Bombus tervestris.

39 secondes pour Io visites pour Bombus lapidarius.

59 secondes pour ro visites pour Bombus agrom

8 o secondes pour ro visites pour Andrena sp.

Iin ce qui concerne les taux de déclenchenent de ces différentes espèces, il m'est difficile de donner des chiffres précis car je n'ai pu suivre qu'un petit nombre d'individus et parce que les différences inter-individuelles sont très grandes.

I,es chiffres extrêmes se situent entre o p. Ioo observé sur roo visites et 8 I p. Ioo sur 43 visites pour Bombus lapidarius.

Entre o p. Ioo observé sur Ioo visites et 25 p. Ioo sur I 60 visites pour Bombus agrorum.

Le seul exemplaire d'Andrena sp. que j'ai pu observer a effectué le déclenchement dans ioo p. Ioo des cas lors de 60 visites. I,es chiffres les plus élevés sont évidemment ceux relatifs à des insectes collecteurs de pollen et à ce propos je dois faire une remarque très importante : plus la saison s'avance, plus il est rare de rencontrer des Bombus collectant du pollen et moins leur efficacité en tant que pollinisatenr est grande. En particulier, à partir du 25 juillet j'ai effectué quelques observations sur des Bombus tervestris $q$ dont le taux de déclenchement oscillait entre o et 2 p. Ioo. Il est sans doute possible d'attribuer ce fait à un ralentissement sérieux ou même à l'arrêt de l'élevage dans les colonies de Bourdon.

Les conséquences de cet arrêt précoce sont certainement graves car à cette époque la I uzenne était encore fleurie et nécessitait la présence de pollinisateurs. Cette baisse du taux de déclenchement est enfin d'autant plus regrettable que le nombre des Bombus présents sur les champs a eu très nettement tendance à augmenter vers cette époque.

C'est ainsi que sur 4 champs étudiés aux environs d'Auneau (Fure-etLoir) les conptages de Bombus ont donné les résultats suivants :

I6 juillet o pour I050 mètres carrés.

I7 juillet o pour I200 mètres carrés.

20 juillet 3 pour $45^{\circ}$ mètres carrés.

2.3 juillet I pour 600 mètres carrés. 
24 juillet o pour 600 mètres carrés.

2.8 juillet I.3 pour 300 mètres carrés.

4 août 20 pour 300 mètres carrés.

() aồt Io pour 300 mètres carrés.

Je ne peux évidemment dire si ces domnées se rapportent à des phénomènes normaux ou si elles sont propres à la localité et à l'année étudiée.

\section{Conclusions.}

I,es populations d'Abeilles domestiques et celles des autres hyménoptères semblent donc très insuffisantes pour assurer une pollinisation satisfaisante de la I,uzerine.

Par aillenrs, il est très important de faire renlarquer qu' en raison sans doute du climat particulièrement chaud et ensoleillé et de la relative abondance des réserves d'eau du sol, la luzerne a été exceptionnellement visitée cette année. En année normale, la luzerne, d'après les dires des apiculteurs, ne serait visitée que dans des régions bien délinitées et il n'y aurait de récoltes de miel de luzerne appréciables que dans des régions encore plus limitées.

Un essai a été tenté pour accroitre la densité en butineuses sur des champs situés dans l'une de ces régions privilégiées. 70 ruches pour 6 hectares, 50 pour + hectares et 20 pour 3 hectares furent placées en bordure de chan1ps de luzerne anx environs d'Auneau (1).

Malheureusement les comptages effectués n'ont pas nis en éviclence une différence significative entre les densités en butineuses de ces champs et des autres champs de cotte mêne région.

Une des raisons tient à ce que le rayon d'action des butineuses, quand les conditions météorologiuues sont aussi favorables, est très grand et que la superficie des champs de luzerne compris dans un rayon de trois kilomètres autour des ruchers était très importante.

Il y a donc en dispersion des butineuses sur une très large superficie, ce qui explique que nalgré le nombre de ruches emplové, il ait été rarenent possible d'observer plus de 2 butineuses au mètre carré. I)ans l'avenir il y aura donc lieu de tenir compte des champs placés an voisinage pour calculer le nombre de ruches nécessaires pour assurer une pollinisation correcte.

Un deuxione point parati important; il s'agit du phénomène de dívive.

J'ai pu vérifier l'assertion des apiculteurs selon laupelle les ruches placées en ligne sur la luzerne dans les plaines de Beauce subissent des pertes importantes à la suite des erreurs d'orientation.

Il est indiscutable que les ruches placées au centre des ruchers se vident de leurs butineuses an profit des ruches placées aux ailes. De ce

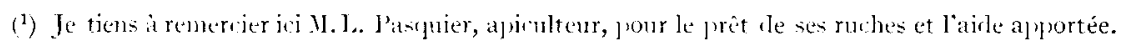


fait l'apiculteur subit des pertes en miel et en colonies par rapport aux ruchers laissés dans les bois à quelques centaines de mètres de là.

I a présence des abeilles en lisière même des champs de I,uzerne étant indispensable pour assurer une pollinisation efficace d'après les auteurs américains (I ECon're, r959), il y a là une contradiction entre les intérêts de l'apiculture et ceux de la production de graines.

Une étude devra donc être entreprise afin de trouver les moyens propres à ellrayer ces phénomènes de dérive et à assurer aux butineuses une possibilité d'orientation correcte.

En ce qui concerne les Hỹménoptères autres que l'A beille, le problème semble dominé par leur rareté relative dans les régions étudiées. Il y aura certainement lieu de chercher à accroître le nombre des individus des espèces reconnutes comme bonnes pollinisatrices.

\section{REFERENCLS BIBIIOGRAPHIQUES}

Lecomte (J.) - - I d1zerne et apiculture. En cours de parutinn dans les Annales de l'A beille. 